Resonant activation in polymer translocation: new insights into the escape dynamics of molecules driven by an oscillating field

This article has been downloaded from IOPscience. Please scroll down to see the full text article.

2010 Phys. Biol. 7034001

(http://iopscience.iop.org/1478-3975/7/3/034001)

View the table of contents for this issue, or go to the journal homepage for more

Download details:

IP Address: 147.163.52.30

The article was downloaded on 01/09/2010 at 10:42

Please note that terms and conditions apply. 


\title{
Resonant activation in polymer translocation: new insights into the escape dynamics of molecules driven by an oscillating field
}

\author{
N Pizzolato ${ }^{1}$, A Fiasconaro ${ }^{2}$, D Persano Adorno ${ }^{1}$ and B Spagnolo ${ }^{1}$ \\ ${ }^{1}$ Dipartimento di Fisica e Tecnologie Relative, Group of Interdisciplinary Physics, Università di \\ Palermo, Viale delle Scienze, edificio 18, I-90128 Palermo, Italy \\ ${ }^{2}$ Departamento de Física de la Materia Condensada, Universidad de Zaragoza, Calle Pedro Cerbuna, \\ 1250009 Zaragoza, Spain \\ E-mail: npizzolato@gip.dft.unipa.it
}

Received 5 February 2010

Accepted for publication 14 July 2010

Published 4 August 2010

Online at stacks.iop.org/PhysBio/7/034001

\begin{abstract}
The translocation of molecules across cellular membranes or through synthetic nanopores is strongly affected by thermal fluctuations. In this work we study how the dynamics of a polymer in a noisy environment changes when the translocation process is driven by an oscillating electric field. An improved version of the Rouse model for a flexible polymer has been adopted to mimic the molecular dynamics, by taking into account the harmonic interactions between adjacent monomers and the excluded-volume effect by introducing a Lennard-Jones potential between all beads. A bending recoil torque has also been included in our model. The polymer dynamics is simulated in a two-dimensional domain by numerically solving the Langevin equations of motion. Thermal fluctuations are taken into account by introducing a Gaussian uncorrelated noise. The mean first translocation time of the polymer centre of inertia shows a minimum as a function of the frequency of the oscillating forcing field. This finding represents the first evidence of the resonant activation behaviour in the dynamics of polymer translocation.
\end{abstract}

\section{Introduction}

The transport of molecules across membranes represents one of the most important processes in biology. In a cell environment, DNA and RNA translocate across nuclear pores and many proteins work on the basis of their ability to go beyond a potential barrier. Cancer target therapy is based on a drug delivery mechanism that crucially depends on the translocation time of the chemotherapeutic molecules $[1,2]$. The study of the transport of macromolecules across a nanopore is also important for technological applications $[3,4]$. In many experimental studies on polymer translocation the passage of a DNA molecule across an $\alpha$-hemolysin $(\alpha$-HL) protein channel is revealed by measuring the reduction of the electrolyte ion current [5-8]. In this way, a linear relationship of the most probable crossing time $\tau_{p}$ with the molecule length was established. Moreover, $\tau_{p}$ scales as the inverse square of the temperature and the dynamics of biopolymer translocation across an $\alpha$-HL channel is found to be governed by pore-molecule interactions. The complex scenario of the translocation dynamics coming from experiments has been enriched by several theoretical and simulative studies [9-15], in which it was shown that the translocation time scales like $\tau_{p} \sim N^{1+2 v}$, where $N$ is the number of monomers and $v$ is the 

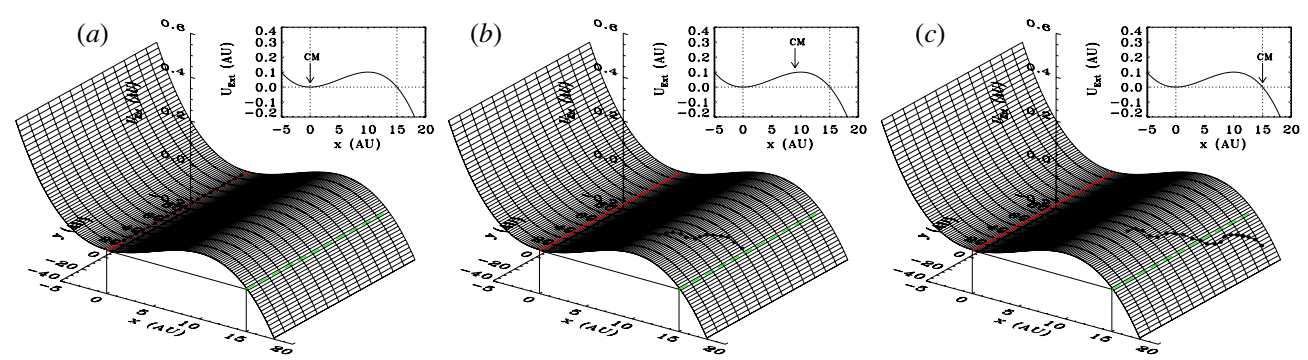

Figure 1. 3D view and projection on the $z-x$ plane of the potential energy $U_{\text {Ext }}$, which is included in our system to simulate the presence of a barrier to be surmounted by the polymer. The three snapshots of a randomly selected event of polymer translocation are shown: the monomers initial configuration (panel $(a)$ ), the crossing polymer (panel $(b)$ ) and the final steric distribution (panel $(c)$ ). The continuous red line and the dashed green line indicate the starting and the finishing line, respectively.

Flory exponent. The translocation process of a chain molecule through a pore in a membrane has been theoretically studied in the presence of a dichotomically fluctuating chemical potential only as a function of its amplitude in [26]. Recent experiments have shown that the application of an ac voltage to drive the translocation process of DNA molecules through a nanopore significantly affects the DNA-nanopore interactions, providing new insights into the DNA conformations [16-20].

The mean time a single Brownian particle takes to cross a potential barrier in the presence of thermal fluctuations, under a periodic forcing field, has been theoretically and experimentally investigated as a function of the driving frequency [21-25]. In this communication, we investigate the role of an external oscillating driving field on the transport dynamics of short polymers starting from a local minimum (metastable state) of the potential landscape and surmounting a barrier in the presence of thermal fluctuations. Molecular dynamics simulations are performed in a two-dimensional domain by modelling the polymer as a flexible chain molecule with adjacent monomers experiencing harmonic interactions. A bending recoil torque is also included in the model and the excluded-volume effect is taken into account by considering a Lennard-Jones (LJ) potential between all beads. Langevin equations of polymer motion are numerically solved by including a Gaussian uncorrelated noise which mimics thermal fluctuations. The mean time the polymer centre of inertia takes to cross the potential barrier for the first time, namely the mean first translocation time (MFTT), changes with the noise intensity. We find a minimum of the MFTT of the molecule as a function of the frequency of the forcing field. This nonlinear behaviour represents, to the best of our knowledge, the first evidence of the resonant activation (RA) phenomenon in polymer translocation. We find that a suitable tuned oscillating field can speed up or slow down the mean time of the translocation process of a molecule crossing a barrier, using the frequency as a control parameter, in accordance with the experimental results reported in [16-20].

\section{The polymer chain model and MD simulations}

The polymer is modelled by a semi-flexible chain of $N$ beads connected by harmonic springs [27]. Both excluded volume effect and van der Waals interactions between all beads are taken into account by introducing a LJ potential. In this work, we investigate the dynamics of a linear chain molecule. In order to confer a suitable stiffness to the chain, a bending recoil torque is included in the model, with a rest angle $\theta_{0}=0$ between two consecutive bonds. In our model any hydrodynamic effect induced by the monomers motion in the solvent is neglected. This approximation implies that our model cannot be used to mimic the molecule behaviour in dilute polymeric solutions, but it appears to be much more appropriate for polymeric melts [28]. The total potential energy of the modelled chain molecule is $U=U_{\mathrm{Har}}+U_{\mathrm{Bend}}+U_{\mathrm{LJ}}$ with

$$
\begin{gathered}
U_{\mathrm{Har}}=\sum_{i=1}^{N-1} K_{\mathrm{r}}\left(r_{i, i+1}-d\right)^{2} \\
U_{\mathrm{Bend}}=\sum_{i=2}^{N-1} K_{\theta}\left(\theta_{i-1, i+1}-\theta_{0}\right)^{2} \\
U_{\mathrm{LJ}}=4 \epsilon_{\mathrm{LJ}} \sum_{i, j(i \neq j)}\left[\left(\frac{\sigma}{r_{i j}}\right)^{12}-\left(\frac{\sigma}{r_{i j}}\right)^{6}\right],
\end{gathered}
$$

where $K_{\mathrm{r}}$ is the elastic constant, $r_{i j}$ is the distance between particles $i$ and $j, d$ is the equilibrium distance between adjacent monomers, $K_{\theta}$ is the bending modulus, $\epsilon_{\mathrm{LJ}}$ is the LJ energy depth and $\sigma$ is the monomer diameter. Polymer translocation dynamics is simulated in a two-dimensional domain. Experiments on the transport dynamics of DNA molecules driven inside an entropic trap array have shown the existence of a quasi-equilibrium state of the polymer during the translocation $[29,30]$. These experimental results suggest the modelization of the polymer translocation as a stochastic process of diffusion in the presence of a potential barrier having the form

$$
U_{\text {Ext }}(x)=a x^{2}-b x^{3}
$$

with the parameters $a=3 \times 10^{-3}$ and $b=2 \times 10^{-4}$, as already adopted in [15]. A three-dimensional view of $U_{\text {Ext }}$ is plotted in figure 1, which includes the three snapshots of the polymer crossing event, showing the monomers starting configuration (figure 1(a)), the crossing polymer (figure 1(b)) and one of the possible final steric distribution (figure 1(c)). The drift of the $i$ th monomer of the chain molecule is described by the following overdamped Langevin equations: 


$$
\begin{gathered}
\frac{\mathrm{d} x_{i}}{\mathrm{~d} t}=-\frac{\partial U_{i j}}{\partial x}-\frac{\partial U_{\mathrm{Ext}}}{\partial x}+\sqrt{D} \xi_{\mathrm{x}}+A \cos (\omega t+\phi) \\
\frac{\mathrm{d} y_{i}}{\mathrm{~d} t}=-\frac{\partial U_{i j}}{\partial y}+\sqrt{D} \xi_{\mathrm{y}},
\end{gathered}
$$

where $U_{i j}$ is the interaction potential between the $i$ th and $j$ th beads given by equations (1)-(3), $U_{\text {Ext }}$ is expressed by equation (4), $\xi_{x}$ and $\xi_{y}$ are the white Gaussian noise modelling the temperature fluctuations, with the usual statistical properties, namely $\left\langle\xi_{k}(t)\right\rangle=0$ and $\left\langle\xi_{k}(t) \xi_{l}(t+\tau)\right\rangle=$ $\delta_{(k, l)} \delta(\tau)$ for $(k, l=x, y)$. $A$ and $\omega$ are respectively the amplitude and the angular frequency of the forcing field and $\phi$ is a randomly chosen initial phase.

We can include the driving force into the interaction potential in such a way that equations (5) and (6) become

$$
\begin{aligned}
\frac{\mathrm{d} x_{i}}{\mathrm{~d} t} & =-\frac{\partial U\left(r_{i j}, \theta_{i j}, t\right)}{\partial x}+\sqrt{D} \xi_{\mathrm{x}_{\mathrm{i}}} \\
\frac{\mathrm{d} y_{i}}{\mathrm{~d} t} & =-\frac{\partial U\left(r_{i j}, \theta_{i j}, t\right)}{\partial y}+\sqrt{D} \xi_{\mathrm{y}_{\mathrm{i}}},
\end{aligned}
$$

where the time-dependent potential is

$$
U\left(r_{i j}, \theta_{i j}, t\right)=U_{i j}+U_{\text {Ext }}-x A \cos (\omega t+\phi) .
$$

Different configurations of the time-dependent potential profile strongly influence the stochastic dynamics of the chain polymer.

In our simulations, the time $t$ is scaled with the friction parameter $\gamma$ as $t=t_{r} / \gamma$, where $t_{r}$ is the real time of the process. The standard LJ time scale is $\tau_{\mathrm{LJ}}=\left(m \sigma^{2} / \epsilon_{\mathrm{LJ}}\right)^{1 / 2}$, where $m$ is the mass of the monomer. A bead of a singlestranded DNA is formed approximately by three nucleotide bases and then $\sigma \sim 1.5 \mathrm{~nm}$ and $m \approx 936 \mathrm{amu}$ [12]. Orders of magnitude of the quantities involved in the process are nanometres for the characteristic lengths of the system (polymer and barrier extension) and microseconds for the time domain. The values of the parameters have been opportunely chosen in order to simulate the translocation process in a realistic general case. A sequence of $10^{3}$ numerical simulations has been performed for different values of the frequency of the forcing field and two values of the noise intensity $D$, namely $D=1.0$ and 4.0. The values of the potential energy parameters are $K_{\mathrm{r}}=K_{\theta}=10, \epsilon_{\mathrm{LJ}}=0.1$, $\sigma=3$ and $d=5$, in arbitrary units (AU). The amplitude of the electric forcing field is $A=5 \times 10^{-2}$ in AU. The number of monomers $N$ is 20. The initial spatial distribution of the polymer is with all monomers at the same coordinate $x_{0}=0$, corresponding to the local minimum of the potential energy of the barrier, and different $y$ coordinates equally spaced by the rest length $d$. Every simulation stops when the $x$ coordinate of the centre of mass of the chain molecule reaches the final position at $x_{f}=15$ (the dashed green line in figure 1).

\section{Results and discussion}

The presence of an oscillating field, driving the polymer crossing dynamics, is experienced by the single monomers as a change of the configuration of the time-dependent potential barrier (equation (9)), which appears periodically lowered and

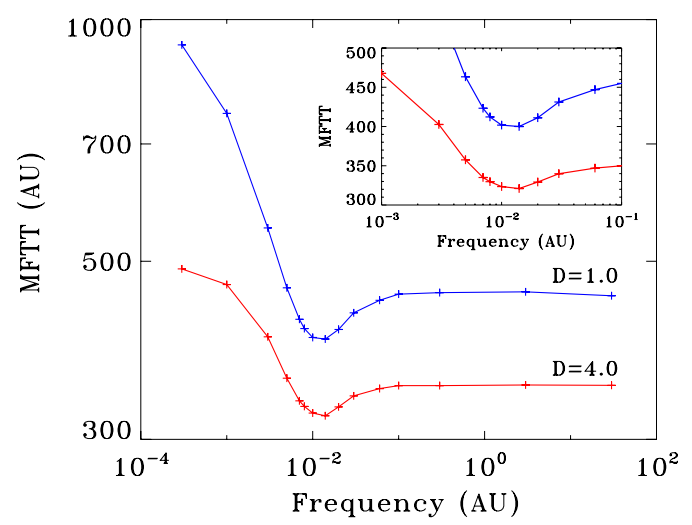

Figure 2. MFTT versus frequency of the forcing field for two different values of the noise intensity $D$. The values of the potential energy parameters are $K_{\mathrm{r}}=K_{\theta}=10, \epsilon_{\mathrm{LJ}}=0.1, \sigma=3$ and $d=5$, in AU. The amplitude of the electric forcing field is $A=5 \times 10^{-2}$ (AU). The number of monomers $N$ is 20 . The inset shows the details of the MFTT versus the frequency characteristic in the RA regime.

heightened. In this view, the frequency of the forcing field, that is the frequency of the oscillating potential, represents a key parameter for the efficacy of the driving mechanism of the polymer translocation process. Our results about the frequency dependence of the MFTT show the three different translocation regimes (figure 2). In the low-frequency domain $\left(\omega<10^{-3}\right)$, the period of the forcing field oscillations is very long with respect to the typical values of the mean crossing time of the chain molecule. In this regime the MFTT is equal to the average of the crossing times over all the initial barrier configurations, because each of these remains nearly unchanged during the entire translocation event. This means that the slower processes critically affect the mean crossing time by increasing its value. As a consequence, we observe long tails in the probability density function (PDF) shown in figure $3(a)$. In the high-frequency domain $\left(\omega>10^{-1}\right)$, a saturation of the translocation time is obtained. In this case, very rapid oscillations of the potential make the molecule chain feel the average potential barrier and, therefore, the MFTT becomes equal to that obtained without any additional periodic driving. For the intermediate frequencies $\left(10^{-2}<\omega<10^{-1}\right)$, the crossing event is strongly correlated with the potential oscillations and the MFTT versus $\omega$ exhibits a minimum at a resonant oscillation rate. This frequency region corresponds to periods of oscillation of the same order of magnitude of the mean time that the polymer takes to cross a static barrier in the lowest configuration of the oscillating potential. In other words, the potential remains around its lowest configuration for enough time to allow the polymer to exit and, even in the case of an initially high or intermediate value of the height of the barrier, the potential can turn into lower configurations within a sufficiently short time lag to facilitate the translocation process. This is the RA phenomenon, firstly studied on a Brownian particle escape dynamics [21-25], but never found previously in molecule dynamics: the polymer, driven by a periodic field oscillating at a period comparable with the characteristic time of the crossing dynamics, reaches the resonant regime that accelerates the translocation process. For each of the frequency values, the thermal noise intensity 

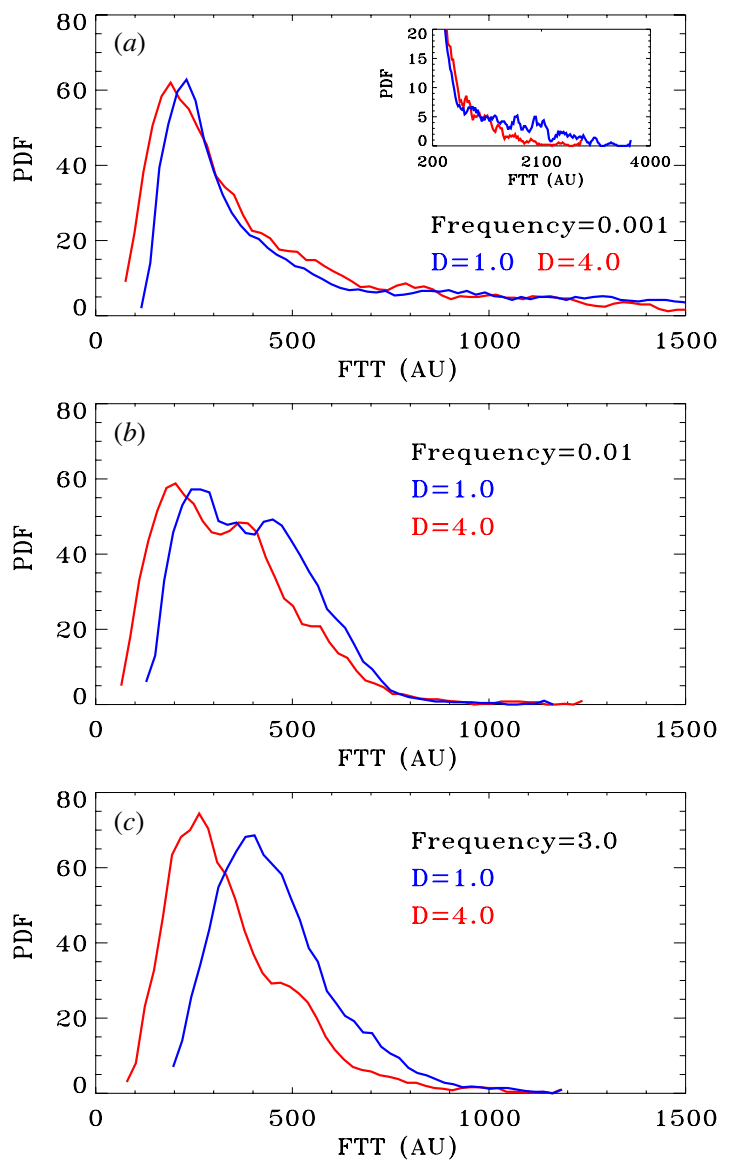

Figure 3. PDF of the FTT. Each panel shows two PDFs, each one characterized by a specific value of the noise intensity, namely $D=1.0$ and 4.0. The three panels differ for the frequency of the forcing field, $\omega=10^{-3}, 10^{-2}$ and 3.0. Panel ( $a$ ) shows the time distribution in the low-frequency region. The long tails (see the inset) indicate that the polymer crosses the potential barrier with a longer mean time. In panel $(b)$ the FTTs are distributed towards shorter values, because of the lowest time scale characterizing the translocation process in the RA regime. Panel (c) shows the probability distribution for the high-frequency domain, where the time scale is the same that is characterized by the presence of a static potential.

$D$ is able to speed up or slow down the crossing process (figure 2), as observed by experiments on the temperature dependence of the translocation time of DNA molecules [7]. The resonant behaviour is not affected by an increase of $D$ and the corresponding translocation dynamics remains unchanged.

The PDF of the first translocation time (FTT) is shown in figure 3 for the three frequency values characterizing the different dynamical domains. Each panel shows two PDFs, each one characterized by a specific value of the noise intensity. In the RA regime (figure $3(b)$ ), the PDFs do not present the long tail at higher crossing times, observed in figure $3(a)$. Consequently, the MFTT reduces its value. The PDF, both at low and high noise intensity, assumes an interesting twopeak structure that suggests the presence of two characteristic times of translocation. We have investigated the translocation times characterizing the two molecular conformations with which the polymer can surmount the barrier, by forming a

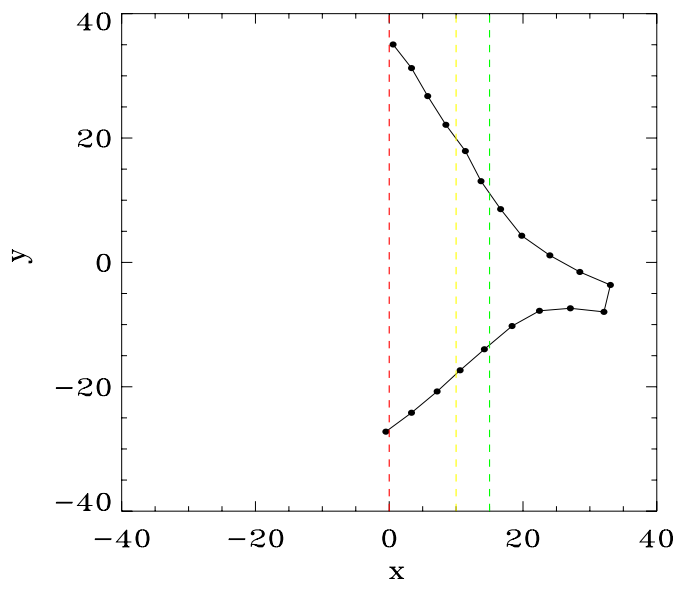

Figure 4. Snapshot of polymer translocation with the hairpin configuration. The red and yellow lines indicate the minimum and maximum of the potential profile, respectively; the green line represents the finishing line of the translocation event.

hairpin or maintaining a linear shape [11, 31], finding that the translocation times of polymers with hairpin conformation are, on average, longer than those characterizing the linear conformation. In particular, by analysing the coordinates of the first, central and last bead of the chain molecule in the final configurations for $D=1.0$ and $\omega=0.01$, we have found that about $67 \%$ of the total configurations, having FTTs in the range of the second maximum in figure $3(b)$, are characterized by hairpin conformations (see figure 4 ). This point, however, deserves further investigations and will be the subject of a forthcoming paper.

In the high-frequency domain (figure 3(c)), the PDFs show the characteristic feature of the static potential case.

\section{Conclusions}

In this work we have investigated the translocation dynamics of short polymers driven by an external oscillating field across a potential barrier, in the presence of thermal fluctuations. We find the first evidence of the RA phenomenon in polymer crossing dynamics. The mean translocation time as a function of the frequency of the driving field shows a nonmonotonic behaviour, with the presence of a minimum, which characterizes the resonant regime. An increase of the thermal noise intensity causes a reduction of the mean crossing times, as observed in experimental works, but leaves the resonant behaviour unchanged. The driving periodic electric field, jointly with the temperature of the system, can speed up or slow down polymer translocation, acting as a tuning mechanism to select a suitable translocation time of the polymer. Moreover, our results show that a driving field which oscillates at a given characteristic frequency can activate the resonant regime of the polymer dynamics and further reduce the mean times of translocation. This effect can be of fundamental importance for all those experiments regarding the cell metabolism, DNA-RNA sequencing and drug delivery mechanism in anti-cancer therapy. 


\section{Acknowledgments}

This work was partially supported by MIUR and CRRNSM, and by the Spanish MICINN through project no FIS200801240, cofinanced by FEDER funds.

\section{References}

[1] Higgins C F 2007 Multiple molecular mechanisms for multidrug resistance transporters Nature 446 749-57

[2] Halwachs S, Schäfer I, Seibel P and Honscha W 2009 Antiepileptic drugs reduce efficacy of methotrexate chemotherapy by downregulation of reduced folate carrier transport activity Leukemia 23 1087-97

[3] Mannion J T, Reccius C H, Cross J D and Craighead H G 2006 Conformational analysis of single DNA molecules undergoing entropically induced motion in nanochannels Biophys. J. 90 4538-45

[4] Sundaresan V B and Leo D J 2008 Modeling and characterization of a chemomechanical actuator using protein transporter Sensors Actuators B 131 384-93

[5] Kasianowicz J J, Brandin E, Branton D and Deamer D W 1996 Characterization of individual polynucleotide molecules using a membrane channel Proc. Natl Acad. Sci. USA 93 13770-3

[6] Akeson M, Branton D, Kasianowicz J J, Brandin E and Deamer D W 1999 Microsecond time-scale discrimination among polycytidylic acid, polyadenylic acid, and polyuridylic acid as homopolymers or as segments within single RNA molecules Biophys. J. 77 3227-33

[7] Meller A, Nivon L, Brandin E, Golovchenko J A and Branton D 2000 Rapid nanopore discrimination between single polynucleotide molecules Proc. Natl Acad. Sci. USA 97 1079-84

[8] Meller A and Branton D 2002 Single molecule measurements of DNA transport through a nanopore Electrophoresis $232583-91$

[9] Lubensky D K and Nelson D R 1999 Driven polymer translocation through a narrow pore Biophys. $J$. 77 1824-38

[10] Storm A J, Storm C, Chen J, Zandbergen H, Joanny J F and Dekker C 2005 Fast DNA translocation through a solid-state nanopore Nano Lett. 5 1193-7

[11] Forrey C and Muthukumar M 2007 Langevin dynamics simulations of ds-DNA translocation through synthetic nanopores J. Chem. Phys. 127015102

[12] Luo K F, Ala Nissila T, Ying S C and Bhattacharya A 2008 Sequence dependence of DNA translocation through a nanopore Phys. Rev. Lett. 100058101
[13] Gracheva M E and Leburton J P 2008 Simulation of electrically tunable semiconductor nanopores for ion current single bio-molecule manipulation J. Comput. Electron. 7 6-9

[14] Panja D and Barkema G T 2008 Passage times for polymer translocation pulled through a narrow pore Biophys. $J$. 94 1630-7

[15] Pizzolato N, Fiasconaro A and Spagnolo B 2009 Noise driven translocation of short polymers in crowded solutions J. Stat. Mech: Theory and Exp. P01011

[16] Deng J, Schoenbach K H, Buescher E S, Hair P S, Fox P M and Beebe S J 2003 The effects of intense submicrosecond electrical pulses on cells Biophys. J. 84 2709-14

[17] Vernier P T, Sun Y, Marcu L, Craft C M and Gundersen M A 2004 Nanoelectropulse-induced phosphatidylserine translocation Biophys. J. 86 4040-8

[18] Sigalov G, Comer J, Timp G and Aksimentiev A 2008 Detection of DNA sequences using an alternating electric field in a nanopore capacitor Nano Lett. 8 56-63

[19] Lathrop D K, Barrall G A, Ervin E N, Keehan M G, Krupka M A, Kawano R, White H S and Hibbs A H 2009 Escape dynamics of DNA from a nanopore under the influence of an AC bias Biophys. J. 96 647a

[20] Nikolaev A and Gracheva M 2009 Controlled DNA translocation through a nanopore membrane with different electrostatic landscapes Biophys. J. 96 649a

[21] Doering C R and Gadoua J C 1992 Resonant activation over a fluctuating barrier Phys. Rev. Lett. 69 2318-21

[22] Bier M and Astumian R D 1993 Matching a diffusive and a kinetic approach for escape over a fluctuating barrier Phys. Rev. Lett. 71 1649-52

[23] Boguna M, Porra J M, Masoliver J and Lindenberg K 1998 Properties of resonant activation phenomena Phys. Rev. E 57 3990-4002

[24] Mantegna R N and Spagnolo B 2000 Phys. Rev. Lett. $843025-8$

[25] Dubkov A A, Agudov N V and Spagnolo B 2004 Phys. Rev. E 69061103

[26] Park P J and Sung W 1998 Polymer translocation induced by adsorption J. Chem. Phys. 108 3013-8

[27] Rouse P E J 1953 A Theory of the linear viscoelastic properties of dilute solutions of coiling polymers J. Chem. Phys. 21 1272-80

[28] Tothova J, Brotovsky B and Lisy V 2005 Single monomer dynamics in DNA polymers Czech. J. Phys. 55 221-7

[29] Han J, Turner S W and Craighead H G 1999 Phys. Rev. Lett. 831688

[30] Han J and Craighead H G 2000 Science 2881026

[31] Wong C T A and Muthukumar M 2008 Scaling theory of polymer translocation into confined regions Biophys. J. $953619-27$ 